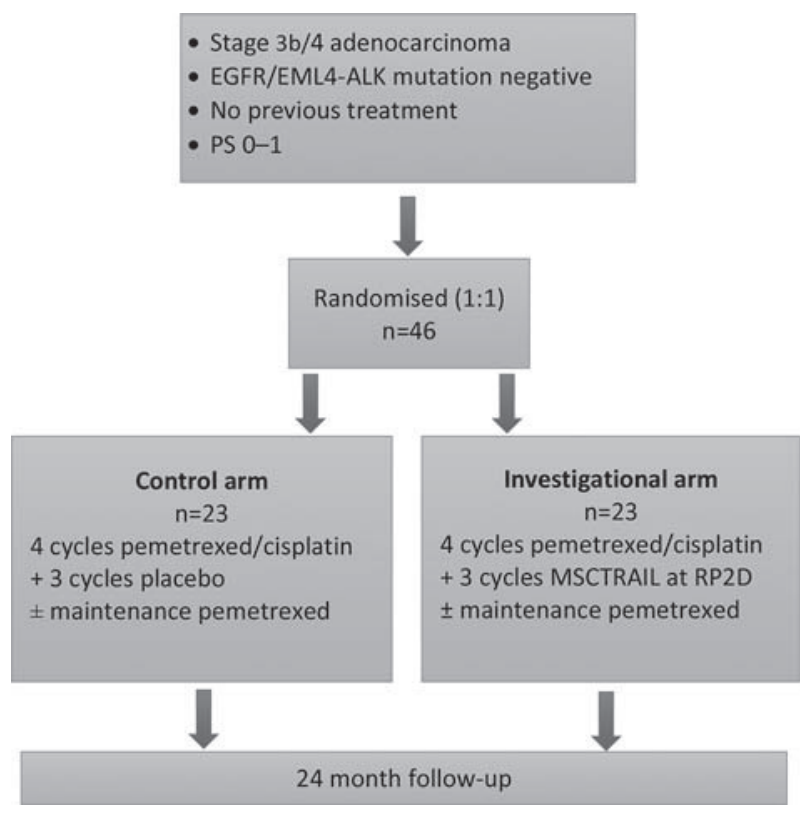

Abstract P256 Figure 1B Phase II Schema.

TRAIL. Successful transduction was confirmed by flow cytometry and cells were subsequently pooled and seeded into a multilayer bioreactor. Once confluent, cells were harvested to form the primary seed stock (PSS). PSS vials underwent further expansion rounds to form a working cell stock (WCC) and subsequent investigational medicinal product (IMP) ready for patient dosing.

Trial Design Patients with stage IIIB/IV adenocarcinoma of the lung (EGFR and EML4-ALK negative) will be eligible for enrolment Phase I is a dose de-escalation study (figure 1A) where patients will receive pemetrexed and cisplatin on day 1 then $4 \times 10^{8}$ MSC-TRAIL cells on day 2 of a 21 day cycle for 3 cycles. Dose limiting toxicities will be recorded. Phase II is a single blinded, randomised, placebo controlled trial consisting of standard chemotherapy and either MSC-TRAIL or placebo (figure 1B). Safety data will be collected and efficacy will be assessed using CT with RECIST (v1.1) criteria at 12 weeks. All patients will be monitored for up to 2 years.

Outcomes Phase I primary outcome is safety and tolerability of MSC-TRAIL.

Phase II is tumour response rate by RECIST ( $\mathrm{v} 1.1$ ) criteria at 12 weeks.

Conclusion We have produced a viable working bank of genetically modified stem cells, If therapy is effective we will plan to expand into larger phase III trials.

\section{P257 ACCEPTABILITY OF A 'VIRTUAL' LUNG NODULE CLINIC TO PATIENTS}

${ }^{1} \mathrm{P}$ Davie, ${ }^{1}$ EA Mackay, ${ }^{2} \mathrm{P}$ Brindas, ${ }^{3} \mathrm{~A}$ Aziz Al Rahim, ${ }^{2} \mathrm{VM}$ Tippett. ${ }^{1}$ University of Liverpool, Liverpool, UK; ${ }^{2}$ University Hospital Aintree, Liverpool, UK; ${ }^{3}$ Blackpool Victoria Hospital, Blackpool, UK

\subsection{6/thoraxjnl-2017-210983.399}

Background and Objectives in 2014 the workload relating to surveillance of indeterminate lung nodules was increasing with associated growing need for clinical and administrative resource. A 'virtual' nodule clinic (VNC) was established with clinicians reviewing scan Results and using an electronic proforma to generate a letter to both GP and patient outlining the results and any follow up plans. With each letter the patients are given details of how to arrange a clinic appointment for face-to-face discussion should they wish, as well as an information leaflet about lung nodules. When details of this novel service were presented at the BTS Winter Conference 2015 colleagues questioned the acceptability of this system to patients.

Methods as many patients will never be seen in clinic face-toface it was not possible to ask their preference directly. As a surrogate we analysed patient records from each VNC between July 2014 and August 2016 to ascertain which patients had been seen in clinic following a VNC review, and whether that appointment was arranged at the patient's request or by a clinician (for example due to nodule growth). If this was at a patient's request we reviewed outpatient letters to ascertain, where possible, the reason for the review.

Results 1591 patient episodes were reviewed. Of these 11 patients requested a face-to-face review. Reasons given included concerns about symptoms, malignancy and stopping follow up too soon. $89 \mathrm{VNC}$ appointments resulted in the clinician requesting an appointment for face-to-face review.

Conclusion The VNC is acceptable to patients with less than $1 \%$ of patient episodes resulting in a request for a face-to-face review. This compares with 5.6\% of patient episodes where the clinician requests an appointment. Reasons given by patients for requesting a review should inform future revision of the patient information leaflet sent with each VNC letter.

\section{P258 CAN WE SAFELY DISCHARGE RESECTED EARLY-STAGE NSCLC FROM THE CLINIC SOONER THAN 5 YEARS?}

${ }^{1} \mathrm{HJP}$ Lurcott, ${ }^{2} \mathrm{SCO}$ Taggart. ${ }^{1}$ University of Manchester, Manchester, UK; ${ }^{2}$ Salford Royal NHS Foundation Trust, Salford, UK

\subsection{6/thoraxjn--2017-210983.400}

Introduction Little guidance is available on the optimal framework for surveillance scanning after surgery for lung cancer. At Salford Royal a CT scan is performed on the anniversary of surgery followed by a further 4 years of scans. Previous audit at the hospital has shown that recurrence rates are low and occur mainly within the first 2 years. It may therefore be possible to discharge patients from the clinic earlier than 5 years. ${ }^{1}$

Methods All patients undergoing surgical resection at Salford Royal from 2005-2013 were identified. Kaplan-Meier survival curves were then drawn with recurrence of disease, with and without metachronous disease, the event of interest. Estimates of recurrence at each year post-surgery were calculated and the log-rank test used to determine the significance of the differences between stages.

Results After exclusions, 211 patients (148 lobectomies; 23 bilobectomies; 20 pneumonectomies; 20 wedge/segmentectomies) were available for analysis. 71 (34\%) patients recurred in the 5 years post-surgery; 57 (80\%) within the chest. 95\% of the 5 year recurrence of stage 1 disease occurred in years 1,2 and $4.94 \%$ and $95 \%$ of the 5 year recurrence in years 1-3 for stage 2 and 3 respectively (figure 1). Significant differences in rates of recurrence were found between stages 1 and $2(p=0.010)$, and stages 2 and $3(p=0.032)$. The rate of metachronous disease for years $1,2,3,4,5$ was $1.6 \% ; 0.7 \%$; $0.8 \% ; 2.7 \%-0.1 \%$ (mean $1.2 \% /$ year) respectively. 
Conclusions This paper supports a move away from the traditional follow-up duration of 5 years by proposing a reduced programme of yearly scans in years 1,2 and 4 for stage 1 disease, and years 1-3 for stage 2 and 3 disease. Patients who are free of disease at this point could be discharged from the clinic accepting a 1.6\% annual rate of metachronous disease. More investigation is warranted on the optimal framework for surveillance within the first 2 years post-surgery.

\section{REFERENCE}

1. Kamalatharan G, Moorcroft C, Shah R, Taggart S. P76 when is it safe to discharge resected stage 1a/1b NSCLC from the clinic? Thorax 2014;69(2):A109-A.

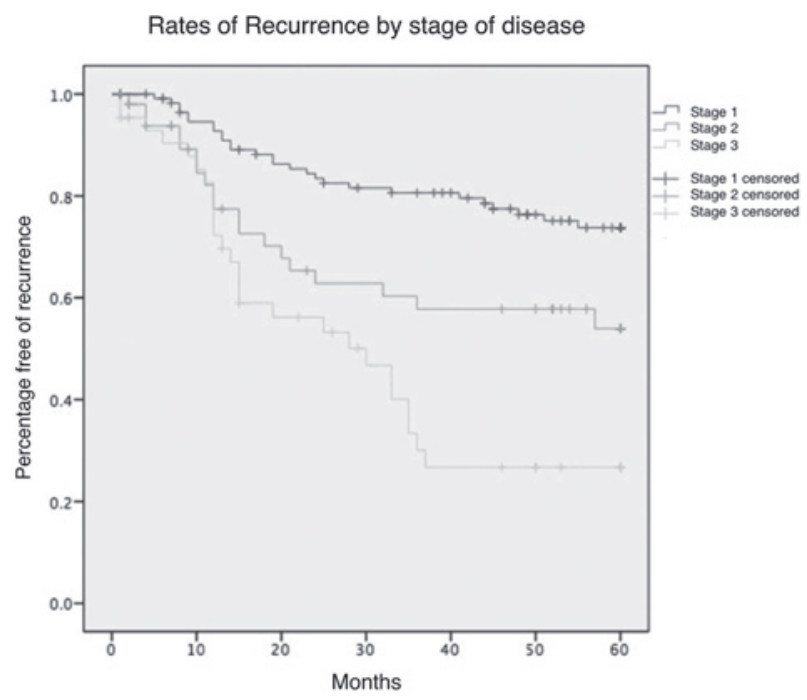

Abstract P258 Figure 1 Graph showing rates of recurrence by stage of disease when metachronous disease was not included as an event of interest.

\section{P259 PURSUIT OF TISSUE: ARE WE DOING PATIENTS A DISSERVICE?}

RM Williams, HE Davies. Llandough University Hospital, Cardiff, UK

\subsection{6/thoraxjnl-2017-210983.401}

Background Whilst pursuit of a histological diagnosis in patients with suspected lung cancer (LC) and good performance status (PS) is indisputable, the advent of novel anti-cancer agents is making us re-examine our approach in patients with poor performance status. NICE guidelines advocate use of anti-cancer therapies in patients with PS $0-1$ (37.1\% of LC patients locally); (NICE 2011); this contrasts with National Lung Cancer Audit recommendations concerning optimal pathological diagnosis rates $(\geq 80 \%)$ (NLCA 2017). Our work evaluates local pathological confirmation rates in patients with poor performance status (3-4) and its impact on patient care. Method All new LC diagnoses over a 12 month period were identified and data collated retrospectively through the CANISC database and electronic record system. Analysis of whether pathological confirmation impacted on the MDT's treatment plan was undertaken.

Results Overall, 277 patients were diagnosed with LC over the 12 month period. 89 patients (32\%) had a PS of 3-4 at diagnosis. The MDT treatment plan for $77 \%$ of this group was specialist palliative care or active monitoring; chemotherapy was recommended for 15 patients -1 received it.
Pathological confirmation was obtained in 38\% of PS 3-4 patients (43\% adenocarcinoma); it influenced management in $43 \%$ of these. Histocytological diagnoses were achieved in 4 patients of PS 4 through a variety of invasive investigations; some unscheduled (pathological fracture fixation), others reflecting diagnostic uncertainty.

Discussion Historically LC patients with a poor performance status received best supportive care and securing a tissue diagnosis was unnecessary. With the advent of personalised treatment and novel therapies, traditional views may need reexamining. Our data demonstrates that, whilst more patients with a poor PS may be considered suitable for anti-cancer therapy, very few receive it. Target driven practice with an unmitigated pursuit of a pathological diagnosis in poor PS patients may be associated with adverse clinical sequelae and waste of valuable resource. Scheduled tests in this population should be considered on an individual basis and involve early MDT discussion. This said, whilst pressure to reach recommended pathological confirmation rates goals remain variables on which hospital LC MDTs are measured; this blanket approach to gaining tissue is likely to continue.

\section{P260 SURVIVAL IMPROVES IN STAGE IV LUNG CANCER PATIENTS}

'B Matata, ${ }^{1} \mathrm{M}$ Shaw, ${ }^{2} \mathrm{~J}$ Maguire, ${ }^{2} \mathrm{M}$ Ledson. ${ }^{1}$ Research Unit, Liverpool Heart and Chest Hospital, Liverpool, UK; ${ }^{2}$ Liverpool Lung Cancer Unit, Liverpool, Anfield, UK

\subsection{6/thoraxjnl-2017-210983.402}

Introduction Liverpool is an area of high socioeconomic deprivation, with more than twice the national incidence of lung cancer. In order to benchmark our survival performance at the Liverpool Lung Cancer Unit (diagnosing about 400 new cases/year) we wanted to compare our Units performance against national figures (32\% 1 year survival, and 10\% 5 year survival). We were also interested in determining if our survival rates had changed over time.

Methods We conducted a retrospective analysis of data for all patients diagnosed over a period of 9 years. All analyses were conducted on the entire dataset stratified on the basis of 3 years' time intervals (2007-2009; 2010-2012; 2013-2015). Demographic data were analysed and compared using descriptive statistics. Survival analysis was conducted by Kaplan Meier survival plots and log- Rank tests. P-values less than 5\% were considered statistically significant.

Results 3710 patients were diagnosed, with a mean age of 71.3 , 52.5\% male. Performance state (PS) $0=15 \%, 1=29.4 \%$, $2=22.2 \%, 3=19.8 \%, 4=6.4 \%$. Stage at diagnosis $1=19.7 \%$, $2=6.6 \%, \quad 3=23.3 \%, 4=41.3 \%$. There was no significant change in numbers, age, PS, histological subtypes and stage over the 3 time periods. However, a survival rate of $40 \%$ and $16 \%$ was observed for 1 year and 5 years respectively, which is higher than the national average (figure 1). In addition, there was an increase in survival for patients diagnosed in latter time period compared with the earlier time periods. Interestingly, only stage IV patients showed significant improvement in survival for 2013-2015 $(\mathrm{p}<0.001)$, a pattern that strongly correlated with an increased oncological treatments (both chemotherapy and radiotherapy) $41.7 \%$ vs $57.0 \%$ $(p<0.001)$. The differences in survival for stage IV patients did not relate with any significant change in age, gender, histological subtype or PS. 